This is the pre print version of the article, which has been published in Acta Neurochirurgica. 2017, vol. 159, no. 5, pp. 907-914. http://dx.doi.org/10.1007/s00701-017-3133-0.

This document has been downloaded from TamPub.uta.fi

a $125(0)$ The Institutional Repository of University of Tampere

\title{
Head Injuries and the Risk of Concurrent Cervical Spine Fractures
}

Tuomo Thesleff, MD

Department of Neuroscience and Rehabilitation, Tampere University Hospital, P.O. Box 2000

FI-33521, Tampere, Finland

Tel:+358-3-311-63023

Fax:+358-3-311-64377

Email: tuomo.thesleff@pshp.fi,

Anneli Kataja, MD

Medical Imaging Centre, Department of Radiology, Tampere University Hospital, P.O. Box 2000

FI-33521, Tampere, Finland

Tel:+358-3-311-611

Fax:+358-3-311-64377

Email: anneli.kataja@pshp.fi

Juha Öhman, MD, PhD

Department of Neuroscience and Rehabilitation, Tampere University Hospital, P.O. Box 2000

FI-33521, Tampere, Finland

Tel:+358-3-311-611

Fax:+358-3-311-64377

Email: juha.ohman@pshp.fi

Teemu M. Luoto, MD, PhD

Department of Neuroscience and Rehabilitation, Tampere University Hospital, P.O. Box 2000

FI-33521, Tampere, Finland

Tel:+358-3-311-66955

Fax:+358-3-311-64377

Email: teemu.luoto@pshp.fi

Corresponding author:

Tuomo Thesleff

Parts of this work were presented as a poster at the 16th European Congress of Neurosurgery in Athens, Greece, in September 2016 and National Neurotrauma Symposium in Santa Fe, New Mexico, USA, in June 2015. 


\begin{abstract}
Background: Cervical spine injuries of variable severity are common among patients with an acute traumatic brain injury (TBI). We hypothesized that TBI patients with positive head CT scans would have a significantly higher risk of having an associated cervical spine fracture compared to patients with negative head CT scans.
\end{abstract}

Methods: This widely generalizable retrospective sample was derived from 3023 consecutive patients who, due to an acute head injury (HI), underwent head CT at the Emergency Department of Tampere University Hospital (August 2010-July 2012). Medical records were reviewed to identify the individuals whose cervical spine was CT-imaged within one week after primary head CT due to a clinical suspicion of a cervical spine injury (CSI) $(n=1091)$.

Results: Of the whole cranio-cervically CT-imaged sample ( $n=1091), 24.7 \%(n=269)$ had an acute CT-positive TBI. Car accidents $22.4 \%(n=244)$ and falls $47.8 \%(n=521)$ were the most frequent injury mechanisms. On cervical CT, any type of fracture was found in $6.6 \%(n=72)$ and dislocation and/or subluxation in $2.8 \%(n=31)$ of the patients. The patients with acute traumatic intracranial lesions had significantly $(\mathrm{p}=0.04 ; \mathrm{OR}=1.689)$ more cervical spine fractures $(9.3 \%, \mathrm{n}=25)$ compared to head CT-negative patients $(5.7 \%, \mathrm{n}=47)$. On an individual cervical column level, head CT positivity was especially related to $\mathrm{C} 6$ fractures $(\mathrm{p}=0.031, \mathrm{OR}=2.769)$. Patients with cervical spine fractures $(n=72)$ had altogether 101 fractured vertebrae which were most often $\mathrm{C} 2(22.8, \mathrm{n}=23), \mathrm{C} 7$ $(19.8 \%, \mathrm{n}=20)$ and C6 (16.8\%, $\mathrm{n}=17)$.

Conclusion: Head trauma patients with acute intracranial lesions on CT have a higher risk for cervical spine fractures in comparison to patients with a CT-negative head injury. Although statistically significant, the difference in fracture rate was small. However, based on these results, we suggest that cervical spine fractures should be acknowledged when treating CT-positive TBIs. Keywords: Brain injuries, Computed tomography, Head injury, Spinal injuries, Cervical spine fractures 


\section{Introduction}

The evaluation of the cervical spine among emergency trauma patients is challenging and is frequently complicated by extracervical injuries and other comorbid conditions [1]. Undetected cervical spine injuries (CSI) may have catastrophic consequences leading to serious neurological impairment or even death [22].

According to the National Emergency X-Radiography Utilization Study (NEXUS), a clinically significant CSI can be excluded without imaging if none of the following five criteria are fulfilled: (i) focal neurological deficits, (ii) midline spinal tenderness, (iii) altered level of consciousness, (iv) intoxication, or (v) distracting injury [11]. However, in many clinical settings, these criteria are not operable. Patients with head injury (HI) and/or traumatic brain injury (TBI) comprise the largest group of patients seen in emergency departments, where clinical examination alone is not sufficient to rule out CSI. The rule of thumb is that all patients with HIs should be treated as if a concomitant CSI is present until proven otherwise. In multitrauma patients, a wholebody computed tomography (CT) from head to pelvis should be obtained on arrival to the

emergency department (ED) [3, 9, 16, 21]. However, patients with less serious or isolated HIs comprise the largest group of patients when it comes to the dilemma of cervical spine clearance. It remains rather unclear which HI patients should undergo routine cervical spine CT-imaging. Excessive and liberal use of CT raises direct medical costs and increases the risk of radiationinduced malignancies [17].

The incidence of CSI in HI patients has been reported to range from 4 to $8 \%[3,4,10,18,20$, 26]. To what extent head trauma severity is associated with concomitant CSIs is controversial and 
depends largely upon the study methods and population studied. Vahldiek et al. did not find an association between positive head CTs and CSIs in their study that comprised only low-energy injuries [25]. Several studies have included only CT-positive or unconscious HI patients [2, 8, 12, 23, 24]. Moreover, many studies have been large multicenter registry, or databank studies in which the details of CSIs and HIs have been limited $[5,8,20]$. In general, several factors in trauma patients have been reported to associate with CSI, for example age, lowered Glasgow Coma Scale score (GCS), injury mechanism, facial fracture, and hypotension $[8,12,20,24]$. Table 1 summarizes the central original publications related to the topic and their key findings.

We hypothesized that HI patients with positive head CT scans would have a significantly higher risk of having a cervical spine fracture than HI patients with negative head CT scans. Due to the suboptimal accuracy of CT in detecting vertebral ligament injuries, our study is focused on fractures.

\section{Insert Table 1 about here}

\section{Materials and methods}

\section{Study design and setting}

This study is a part of the Tampere Traumatic Head and Brain Injury Study. The patient sample in the registry includes all consecutive patients $(n=3023)$ with HI who underwent head CT at the Tampere University Hospital's ED between August 2010 and July 2012. The patients were prospectively enrolled from the ED and the data were retrospectively recorded.In the study, the minimum diagnostics criteria for TBI were based on the WHO Neurotrauma Task Force recommendation[13]. Referral criteria for acute head CT were based on the former Scandinavian 
guidelines for initial management of minimal, mild and moderate head injuries [14]. Ethics approval (code: R10027) for the study was obtained from the ethical committee of the Pirkanmaa Hospital District in Tampere, Finland.

\section{Clinical data}

Data collected from the registry included subject- and injury-related data, clinical information from the ED, and data on neurosurgical interventions. Also, the mechanisms of injury and time intervals (injury - ED admission - head CT - ED discharge) were recorded. Destination after the ED was categorized into four groups: home, hospital ward, local health center, or death. In retrospect, the medical records of all these patients $(n=3023)$ were carefully reviewed to select those individuals whose cervical spine was CT-imaged due to a clinical suspicion of a CSI within one week after primary head CT. Cervical CT was performed primarily according to the NEXUS recommendations [11]. On arrival, multitrauma patients underwent whole-body CT (comprising cervical spine) according to international recommendations $[3,9,16,21]$. A total of $1091(36.1 \%)$ cervical spine CT-imaged patients were identified and included into the current study. The majority of the patients $(96.5 \%, \mathrm{n}=1053)$ were cervically CT-imaged within 24 hours after primary head CT and the rest in one to four days time. Of the CSI patients, the presence of possible spinal cord injury and radiculopathy as well as the American Spinal Injury Association (ASIA) scores were recorded [15]. Medical records from a period of one year post-injury were reviewed to collect information on possible cervical spine surgery due to the index injury.

\section{Imaging data}

In the ED, an emergency non-contrast head CT scan was performed as per Scandinavian guidelines for all patients, using a 64-row CT scanner (Lightspeed VCT; GE, Wisconsin, USA)[14]. In a nonon-call setting, all head CT scans were analyzed and systematically coded by two neuroradiologists 
using a structured data collection form. Acute traumatic intracranial lesions included subdural hematoma and effusion (SDH), epidural hematoma and effusion (EDH), diffuse axonal injury (DAI) lesions, edema, compression of the cerebrospinal fluid spaces, midline shift, contusions, pneumocephalus, skull fracture, and traumatic subarachnoid hemorrhage.

Cervical CT imaging was performed with the same scanner as for the head CTs. CSI was defined as a fracture or subluxation of any of the cervical vertebrae. Whiplash injuries without radiological findings were not included in the analysis. The injured cervical spine level, including occipital condyle ( $\mathrm{C} 0)$ fracture, together with a detailed anatomic description of each vertebra and CT-detectible ligament injury, was recorded systematically by the first author (T.T.). On clinical basis, magnetic resonance imaging was performed on the patients with spinal cord injury, but their results were not analyzed in this study.

\section{Statistical analyses}

The normality of the variable distributions was tested using the Kolmogorov-Smirnov and ShapiroWilk tests. Continuous variables were analyzed with the Pearson (normal distribution) and Spearman (skewed distribution) correlation coefficients. Group comparisons were tested with the Student's t-test (normal distribution) and the Mann-Whitney U-test (skewed distribution).

\section{Results}

\section{Characteristics of the study sample}

The main clinical characteristics of the study sample $(n=1091)$ are presented in Table 2a and CSIrelated characteristics in Table $2 \mathrm{~b}$. For comparison, the characteristics of the patients with positive head CT scans $(n=269)$ are presented parallel with the patients with negative head CT scans $(n=822)$ and the whole study sample $(n=1091)$. Of all the patients $(n=1091), 607(55.6 \%)$ fulfilled 
the clinical criteria for mild TBI, $201(18.4 \%)$ had a moderate or severe TBI and the rest $(\mathrm{n}=283$, $25.9 \%$ ) having a head trauma without clear signs of TBI. Cervical spinal cord injury was found in $13(16.9 \%)$ of the CSI patients. In one patient, the existence of a possible spinal cord injury remained unknown due to early death caused by a severe TBI.

Insert Table $2 a$ and $2 b$ about here

\section{Cervical CT findings}

On cervical CT, CSI was found in $7.1 \%(n=77)$ and cervical spine fracture in $6.6 \%(n=72)$ of the patients $(\mathrm{n}=1091)$. In total, these patients sustained 101 fractured vertebrae. Five patients $(0.5 \%)$ had a CSI with only a CT-detectible ligament injury. Of the patients with CSI, $31(40.3 \%)$ had dislocations and/or subluxations. The mean dislocation was three millimeters (range 1 - 10mm). The distribution of fractures and dislocations/subluxations is presented in Figure 1.

Insert Figure 1 about here

\section{Cervical spine fractures in patients with CT-positive versus CT-negative head injuries}

Patients with CT-positive acute traumatic intracranial lesions $(9.3 \%, \mathrm{n}=25)$ had significantly more cervical spine fractures (Pearson chi-square, $\mathrm{p}=0.04 ; \mathrm{OR}=1.689,95 \% \mathrm{CI}=1.019-2.802$ ) compared with head CT-negative HI patients $(5.7 \%, \mathrm{n}=47)$. Interestingly, patients with positive head CT scans had fewer spinal cord injuries $(0.4 \%, \mathrm{n}=1$ compared with $1.5 \%, \mathrm{n}=12$; statistical comparison not done due to small group sizes). Moreover, positive head CT findings had significant association 
with C6 vertebra fractures $(3.0 \%, \mathrm{n}=8$ versus $1.1 \%, \mathrm{n}=9 ; \mathrm{p}=0.031, \mathrm{OR}=2.769,95 \% \mathrm{CI}=1.057$ 7.250), but not with other cervical vertebra fractures ( $\mathrm{C} 0-5$ and $\mathrm{C} 7)$ or dislocations/subluxations alone. The distribution of fractures between the upper (C0-C2) and lower (C3-C7) cervical spine did not differ between the two groups. Patients with positive head CT scans $(2.2 \%, n=6)$ did not have more neurosurgical operations within one year post-HI due to cervical spine fractures compared with patients with negative head CT scans $(2.8 \%, \mathrm{n}=23 ; \mathrm{p}=0.614)$.

\section{Risk of Cervical Spine Fractures in Relation to Neurological Picture and Mechanism of Injury}

Patients with cervical spine fractures had more frequently moderate to severe TBIs $(22.2 \%, \mathrm{n}=16$ vs. $18.2 \%, \mathrm{n}=185)$ but the difference did not reach statistical significance $(\mathrm{p}=0.376)$. Also, GCS levels $(\mathrm{p}=0.464)$, and number of patients with loss of consciousness $(15.3 \%, \mathrm{n}=11$ vs. $23.7 \%$, $\mathrm{n}=241, \mathrm{p}=0.264)$ were similar between the groups. However data on GCS levels were missing in $30.6 \%(n=334)$, and on patients with loss of consciousness in $42.4 \%(n=463)$. There were no statistically significant differences in the gender distribution (males $73.6 \%, \mathrm{n}=53$ vs. $64.9 \%, \mathrm{n}=661$, $\mathrm{p}=0.132)$ or mechanism of injury when classified as in Table $2 \mathrm{a}(\mathrm{p}=0.352)$. However, Patients with fractures were longer times hospitalized (median 96.4 hours vs. 21.3 hours, $\mathrm{p}<0.001$ ) and more neurosurgical procedures $(19.4 \%, \mathrm{n}=37$ vs. $7.9 \%, \mathrm{n}=81, \mathrm{p}<0.001)$ were performed on them compared to patients without cervical spine fractures.

\section{Discussion}

For clinicians working in the ED setting, detecting patients with a possible CSI is of the utmost importance. Acute $\mathrm{HI}$ is one of the most common causes of ED admissions, and the failure to detect CSI, especially in patients with a decreased level of consciousness, can have catastrophic 
consequences. We found that HI patients with acute intracranial lesions on CT had almost a twofold risk of cervical spine fractures in comparison to CT-negative head trauma patients. Especially, C6 fractures were associated with head CT positivity. Because of the suboptimal accuracy of CT in detecting ligament injuries, we concentrated mainly on fractures in the risk analysis. Compared to prior studies, our results might have superior generalizability as our sample is non-selected and consists of HIs ranging in severity from minimal to severe.

In contrast to our results, there are several reports in the literature stating that $\mathrm{HI}$ is not an independent risk factor for CSI $[6,8,23,25,26]$. Some of the incoherence between the reported coincidence of TBI and CSI may derive from the differences in the study populations and methods used in different studies. For example, Hasler and co-researchers studied an extensive injury databank and reported that lowered GCS scores predicted CSI, but they found no association between severe HI and CSI [8]. However, Michael and co-researchers found an association between severe HI and CSI in a study conducted in an individual major trauma hospital [18].

We showed that patients with CT-positive TBI not only have more cervical spine fractures, but, in addition, they have significantly more C6 vertebra fractures. However, the fracture distribution in regard to upper and lower cervical spine did not differ significantly between these two groups (head CT positive versus negative). Moreover, the incidence of cervical vertebra dislocations/subluxations or neurosurgical operations due to cervical spine fracture was not elevated in the CT-positive TBI group. A neurosurgical operation due to cervical spine fracture was performed on $37.7 \%(n=29)$ of the CSI patients during their first year post-injury. None of the patients were treated with a halo-vest, and a few of the patients were treated with either a soft or rigid collar (data not shown). In the light of our findings, it can be speculated that, although head $\mathrm{CT}$ positivity is associated with an increased risk of cervical spine fractures, these fractures are 
equally severe clinically as measured by the need for surgery. Interestingly, there were also fewer cervical spinal cord injuries in CT-positive TBI patients. This may be due to differences in injury mechanisms. Injury mechanism among patients with a spinal cord injury was remarkably often a simple ground level fall $(\mathrm{n}=6,46.2 \%)$, whereas among patientswithout a spinal cord injury ground level falls accounted for only $29.6 \%(n=319)$ of the cases. Moreover, patients with a spinal cord injury were older (median age 65.9 versus 47.0 years) than patients without a spinal cord injury. It may be speculated that falling accidents of elderly people are more likely to cause sole cervical spine injuries than CT-positive craniocervical injuries. In a previous study of more than 5000 trauma patients, Williams and coworkers found a negative association between TBI and spinal cord injury, which is in line with our findings [26]. In order to identify risk factors for cervical spine fractures, we compared patients with a fracture to patients who did not have a fracture. In contrast to majority of the prior studies, we could not find association between neurological status and fracture risk $[8,12,24,26]$. However, the number of missing data on GCS levels and loss of consciousness in our series is considerably high and prevents drawing a reliable conclusion in this regard. Injury mechanisms were also somewhat similar between the groups. Ground level falls accounted for about one third of the cases in both groups and car accidents were more frequent in the fracture group $(30.6 \%, n=22$ vs. $21.8 \%, n=222)$. However, difference in injury mechanisms was not statistically significant. Nevertheless, it is widely accepted that high energy injury mechanism increase the risk of cervical spine fractures.

Our results on the distribution of cervical spine fractures were mostly in concordance with the previous reports on trauma patients, although the number of studies focusing on HI patients is scarce[7]. In our study, 30.6\% $(n=22)$ of the cervical fracture patients had a multilevel injury. C2 (including odontoid) was the most commonly fractured vertebra consisting $22.8 \%$ ( $n=23$ ) of all $(n=101)$ fractured individual vertebrae. Subaxial fractures $(n=64)$ consisted $63.4 \%$ of all fractured vertebrae. Vertebrae C6 $(n=17,16.8 \%)$ and C7 $(n=20,19.8 \%)$ were the most commonly fractured 
subaxial vertebrae, whereas $\mathrm{C} 3$ was the vertebra least likely to be injured $(\mathrm{n}=6,5.9 \%)$. Dislocations and subluxations occurred most frequently in C1/C2 (n=9, 29\%) and C4/C5 (n=7, 22.6\%) levels. In the study by Goldberg and co-authors on the distribution and patterns of blunt CSIs, the findings were slightly different compared to our results [7]. In line with our results, Goldberg et al. reported $\mathrm{C} 2$ to be the most commonly fractured cervical vertebra. On the contrary, they found C6 to be the most common site of subaxial fracture, whereas in our series the most common site was C7 followed by C6 fractures. Different study populations (blunt trauma in the study by Goldberg and coworkers versus HI patients in our study) and different injury coding habits may explain the small differences in the fracture distribution.

Our study represents a large population-based HI sample that is commonly seen in EDs internationally. The sample includes a wide severity spectrum of HIs that were treated at the ED at varying time delays post-injury. Although not unique, our findings support the prior studies on the association between TBI and CSI $[3,10,12,18]$. However, the main finding of our study (increased risk of cervical spine fractures among head CT-positive TBI patients) must be interpreted with caution given the subtle difference in fracture rate that is only slightly statistically significant $(\mathrm{p}=0.04)$. The main limitation of our study is the retrospective nature, and therefore the number of missing data (e.g. GCS and alcohol consumption) is relatively large, which may influence the main conclusions. Secondly, head CT criteria in minimal, mild and moderate HIs in the ED were based on the Scandinavian guidelines. Apparently, some patients in the sample did not fulfill the Scandinavian CT criteria and were CT-imaged without solid indications. In contrast, possibly some HI patients did not undergo CT imaging although they met the criteria. Thirdly, cervical ligament, and soft tissue injuries may have been missed since patients were not systematically assessed with MRI. However, the main emphasis of this study was on fractures. Most probably, clinically significant CSIs were not missed since our study included patients whose cervical spine was CT- 
imaged up to one week post-injury and the care of CSIs in the study catchment area is centered to our hospital.

\section{Conclusion}

Head trauma patients with acute intracranial lesions on CT have higher risk for cervical spine fractures in comparison to patients with a CT-negative head injury. However, these fractures did not cause more spinal cord injuries or require more neurosurgical spinal intervention compared to head CT-negative cervical spine fractures. Although statistically significant, the difference in fracture rate is small (9.3\% versus 5.7\%). Nevertheless, cervical spine fracture should be acknowledged when treating CT-positive TBI patients. CT imaging of the cervical spine in the case of CT-positive TBI should be considered based on these findings.

\section{Acknowledgements}

The authors wish to thank nurse Anne Simi for her assistance in data collection.

\section{Conflict of interest}

All authors certify that they have no affiliations with or involvement in any organization or entity with any financial interest (such as honoraria; educational grants; participation in speakers' bureaus; membership, employment, consultancies, stock ownership, or other equity interest; and expert testimony or patent-licensing arrangements), or non-financial interest (such as personal or 
professional relationships, affiliations, knowledge or beliefs) in the subject matter or materials discussed in this manuscript.

\section{Funding}

No funding was received for this work.

\section{Ethical approval}

For this type of study formal consent is not required.

\section{References}

(1)Anderson PA, Muchow RD, Munoz A, Tontz WL, Resnick DK (2010) Clearance of the asymptomatic cervical spine: a meta-analysis. J Orthop Trauma 24:100-106

(2)Bayless P, Ray VG (1989) Incidence of cervical spine injuries in association with blunt head trauma. Am J Emerg Med 7:139-142

(3)Blackmore CC, Emerson SS, Mann FA, Koepsell TD (1999) Cervical spine imaging in patients with trauma: determination of fracture risk to optimize use. Radiology 211:759-765

(4)Drainer EK, Graham CA, Munro PT (2003) Blunt cervical spine injuries in Scotland 1995-2000. Injury 34:330-333

(5)Fujii T, Faul M, Sasser S (2013) Risk factors for cervical spine injury among patients with traumatic brain injury. J Emerg Trauma Shock 6:252-258

(6)Gbaanador GB, Fruin AH, Taylon C (1986) Role of routine emergency cervical radiography in head trauma. Am J Surg 152:643-648

(7)Goldberg W, Mueller C, Panacek E, Tigges S, Hoffman JR, Mower WR, NEXUS Group (2001)

Distribution and patterns of blunt traumatic cervical spine injury. Ann Emerg Med 38:17-21

(8)Hasler RM, Exadaktylos AK, Bouamra O, Benneker LM, Clancy M, Sieber R, Zimmermann H, Lecky F (2012) Epidemiology and predictors of cervical spine injury in adult major trauma patients: a multicenter cohort study. J Trauma Acute Care Surg 72:975-981

(9)Hessmann MH, Hofmann A, Kreitner KF, Lott C, Rommens PM (2006) The benefit of multislice CT in the emergency room management of polytraumatized patients. Acta Chir Belg 106:500-507

(10)Hills MW, Deane SA (1993) Head injury and facial injury: is there an increased risk of cervical spine injury?. J Trauma 34:549-53; discussion 553-4

(11)Hoffman JR, Wolfson AB, Todd K, Mower WR (1998) Selective cervical spine radiography in blunt trauma: methodology of the National Emergency X-Radiography Utilization Study (NEXUS). Ann Emerg Med 32:461-469

(12)Holly LT, Kelly DF, Counelis GJ, Blinman T, McArthur DL, Cryer HG (2002) Cervical spine trauma associated with moderate and severe head injury: incidence, risk factors, and injury characteristics. J

Neurosurg 96:285-291 
(13)Holm L, Cassidy JD, Carroll LJ, Borg J, Neurotrauma Task Force on Mild Traumatic Brain Injury of the WHO Collaborating Centre (2005) Summary of the WHO Collaborating Centre for Neurotrauma Task Force on Mild Traumatic Brain Injury. J Rehabil Med 37:137-141

(14)Ingebrigtsen T, Romner B, Kock-Jensen C (2000) Scandinavian guidelines for initial management of minimal, mild, and moderate head injuries. The Scandinavian Neurotrauma Committee. J Trauma 48:760766

(15)Kirshblum SC, Burns SP, Biering-Sorensen F, Donovan W, Graves DE, Jha A, Johansen M, Jones L, Krassioukov A, Mulcahey MJ, Schmidt-Read M, Waring W (2011) International standards for neurological classification of spinal cord injury (revised 2011). J Spinal Cord Med 34:535-546

(16)Linsenmaier U, Krotz M, Hauser H, Rock C, Rieger J, Bohndorf K, Pfeifer KJ, Reiser M (2002) Wholebody computed tomography in polytrauma: techniques and management. Eur Radiol 12:1728-1740

(17)Mathews JD, Forsythe AV, Brady Z, Butler MW, Goergen SK, Byrnes GB, Giles GG, Wallace AB,

Anderson PR, Guiver TA, McGale P, Cain TM, Dowty JG, Bickerstaffe AC, Darby SC (2013) Cancer risk in 680,000 people exposed to computed tomography scans in childhood or adolescence: data linkage study of 11 million Australians. BMJ 346:f2360

(18)Michael DB, Guyot DR, Darmody WR (1989) Coincidence of head and cervical spine injury. J Neurotrauma 6:177-189

(19)Milby AH, Halpern CH, Guo W, Stein SC (2008) Prevalence of cervical spinal injury in trauma. Neurosurg Focus 25:E10

(20)Mulligan RP, Friedman JA, Mahabir RC (2010) A nationwide review of the associations among cervical spine injuries, head injuries, and facial fractures. J Trauma 68:587-592

(21)Schmidt OI, Gahr RH, Gosse A, Heyde CE (2009) ATLS(R) and damage control in spine trauma. World J Emerg Surg 4:9-7922-4-9

(22)Schoenfeld AJ, Bono CM, McGuire KJ, Warholic N, Harris MB (2010) Computed tomography alone versus computed tomography and magnetic resonance imaging in the identification of occult injuries to the cervical spine: a meta-analysis. J Trauma 68:109-13; discussion 113-4

(23)Soicher E, Demetriades D (1991) Cervical spine injuries in patients with head injuries. Br J Surg 78:1013-1014

(24)Tian HL, Guo Y, Hu J, Rong BY, Wang G, Gao WW, Chen SW, Chen H (2009) Clinical characterization of comatose patients with cervical spine injury and traumatic brain injury. $\mathrm{J}$ Trauma 67:1305-1310

(25)Vahldiek JL, Thieme S, Hamm B, Niehues SM (2016) Incidence of combined cranial and cervical spine injuries in patients with blunt minor trauma: are combined CT examinations of the head and cervical spine justified?. Acta Radiol

(26)Williams J, Jehle D, Cottington E, Shufflebarger C (1992) Head, facial, and clavicular trauma as a predictor of cervical-spine injury. Ann Emerg Med 21:719-722

\section{Figure legends}

Fig. 1a The distribution of cervical spine fractures

Fig. 1b The distribution of cervical spine dislocations/subluxations. 
